\title{
An Electrode Setup for Non-contact Dielectric Response Measurement
}

\author{
J. Hao ${ }^{1}, X . \mathrm{Xu}^{2}$, N. Taylor ${ }^{1}$ \\ 1 Electromagnetic Engineering, KTH Royal Institute of Technology, SE-100 44 Stockholm, Sweden \\ 2 Electric Power Engineering, Chalmers University of Technology, SE 412-96 Gothenburg, Sweden
}

\begin{abstract}
The dielectric response measurement is a widely used technique for characterizing dielectric materials. However, the contact problems between samples and electrodes existing in the use of conventional electrode setup limit the accuracy of the measurement. This paper studies an electrode arrangement that avoids direct contacts of the sample with the electrodes on both sides. The edge effect of this arrangement is calculated by the FEM model. The equations to derive the complex permittivity are presented. The measurement instrument is described and the influence of the small voltage between its measure and guard terminals is analyzed. The error sensitivities of the non-contact and contact methods are compared. The results show that this electrode arrangement can be used to perform non-contact measurements, the edge effect of it is not significant, and the guard voltage is not an obstacle to obtain results with high accuracy. Overall, the non-contact electrode arrangement combined with the IDAX 300 can potentially improve the accuracy of dielectric response measurements although the noncontact methods can increase the sensitivities to errors.
\end{abstract}

\section{Introduction}

Dielectric response measurement is a popular technique to characterize the properties of dielectric materials. The dielectric constant and loss of insulating samples at different frequencies is obtained by frequency domain spectroscopy (FDS) measurements.

The conventional way to perform the FDS measurements is to place the sample between two conductors or electrodes, and apply a voltage with different frequencies on the electrodes. There are several sources of inherent errors in the conventional electrodes which can affect both the reproducibility and the accuracy of the results. One main problem is related to the pressure applied by the electrodes on the sample. Whether applying the overloaded or the insufficient potential will lead to contact problems, affecting the accuracy of the results [1]. Another problem is the reactions between the sample and the metal electrodes. The reactions can increase the number of mobile charge carriers, leading to conductivity beyond the level of the sample's intrinsic behavior. For example, the atoms in the electrode made by $\mathrm{Cu}$ can diffuse into the polyimide to form clusters [2].

There are several types of electrode arrangements that are aimed at solving the problems of conventional electrodes. Mercury electrode [3] is one of choices, which can assure satisfactory contacts between the sample and electrodes.
However, mercury is toxic, and the properties of the sample can be changed due to the chemical reactions. Another alternative is called foil electrodes, where a sheet of foil is adhered to either side of the sample by a film of wax or petrolatum [4]. The foil electrode can decrease the contact problem, and eliminate the error due to the field lines emitted or received by the vertical surfaces of the electrodes. However, the reusability and the edge correction are considerable. Apart from these, the conducting painted or sputtered or evaporated electrodes are also concerned, which consist of a coating of conducting material on either side of the sample [5]. They can establish intimate contacts between samples and electrodes. However, the coating of the deposition materials with sufficient resistance of may affect the power factor, and the preparation is time-consuming. In addition to these, the presence of other chemicals from paint can be a problem. Similarly, the conductive rubber can improve the contact conditions between the sample and the electrodes, but the resistance of the rubber can not be neglected. The electrodes discussed above are aimed at eliminating the unsatisfactory contact conditions, but they are not helpful for avoiding the reactions between the sample and electrodes. For the latter, a type of electrodes with an insulating layer on each electrode is introduced, which is called the blocking electrode. The insulating layers can effectively block the reactions between the sample and the air, but the insulating films need to be carefully compensated with high precision in order to obtain true dielectric constant of the sample [6].

Among these electrodes, a type of electrode with the sample placed on the bottom electrode and separated by an air gap [1] has attracted some attention. The sensitivity of the error sources [7], the correction of the geometric influence [8, 9], and the application of this electrode [10] have been analyzed account for using this method to determine the permittivity of insulating materials. However, the ideal contact-free situation has not been realized as the sample still needs contact with the bottom electrode which will also lead to surface resistance and contact problems if the sample is not plane. In addition, the contact quality will become worse as the lack of an appropriate pressure from the upper electrode.

Based on this idea, one type of electrode arrangement that can avoid direct contacts of the sample with both the bottom and the top electrode is introduced. The edge effect of this arrangement is calculated by the FEM model. The measurement instrument is described and the influence of the potential difference between two terminals of IDAX input is analyzed. The error sensitivities are compared between the non-contact and contact methods 


\subsection{Electrode arrangement}

In order to realize the non-contact measurement, one crucial technique is to find a way to fix the sample between the top and the bottom electrode without producing contacts between them. For this purpose, one type of electrode is designed as shown in Figure 1. This arrangement consists of a measuring electrode and a high-voltage electrode made by stainless steel, which have thickness of $5 \mathrm{~mm}$. The main difference from the conventional electrodes is that both of the measuring electrode and the highvoltage electrode have guard rings, and each electrode with its own guard electrode is fixed in a flat baseplate which is made by plastic insulating materials. In the measurements, the highvoltage electrode and its guard ring are both connected to the output of the measuring instrument, and the measuring electrode and its guard electrode are connected to the input of the instrument and ground separately. The height of the guard electrode relative to the baseplate can be adjusted to be higher than the measuring/high-voltage electrode by inserting thin films between the baseplate and the guard electrode. In this way, the sample can be clamped by the two guard electrodes from the opposite directions, and assure an air gap between the measuring/high-voltage electrode and the sample.

The baseplates with the electrodes are supported and fixed by two Y-shaped frames made by wood. The top and the bottom electrodes are resembled by screws through the wood frame and then fixed by the nuts. The pressure applied to the sample can be adjusted by changing the length of the springs. If the sample is soft, the sample may droop under the effect of the gravity, leading to changes in air gap distances or unwanted contacts between the sample and measuring/high-voltage electrodes. To solve this problem, the whole electrode arrangement can be rotated 90 degrees and keep stable with the help of the Y-shape frame. In this case, the sample is held vertically, and the gravity will hardly change the distance between the vertically placed sample and measuring/high-voltage electrodes.

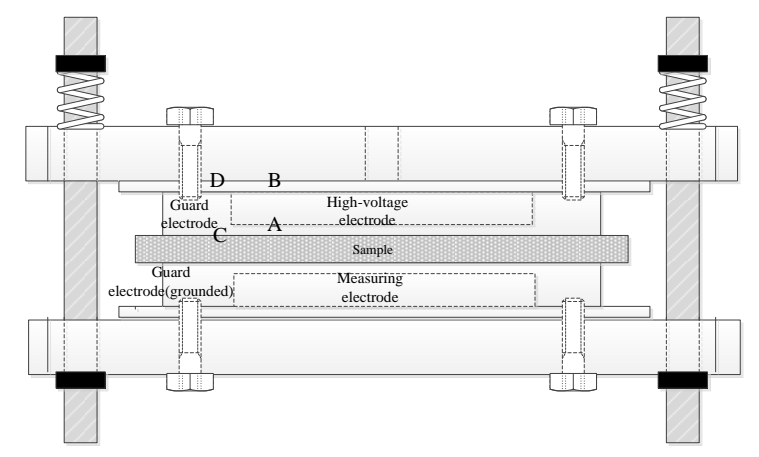

Figure 1. Sketch of the contact-free electrode arrangement

\subsection{Measurement Setup}

The instrument used to perform the dielectric response measurement is the IDAX300, which can export an output voltage with different frequencies and measure the resulting current through the sample. The claimed relative errors in the manual are less than $5 \%+0.0002 \mathrm{~F}$ from $45 \mathrm{~Hz}$ to $70 \mathrm{~Hz}$ for the capacitance that is over $300 \mathrm{pF}, 1 \%+0.0003 \mathrm{~F}$ from $1 \mathrm{mHz}$ to $100 \mathrm{~Hz}$ for capacitance that is over $1000 \mathrm{pF}$ and $2 \%+0.0005 \mathrm{~F}$ from $100 \mathrm{~Hz}$ to $1 \mathrm{kHz}$ for capacitance that is over $1000 \mathrm{pF}$.

\subsection{Air reference Method}

In this paper, the air reference method is used to eliminate the influence of system errors [1]. For each sample, the IDAX measures the complex capacitance twice with and without the sample placed between the high-voltage electrode and the measuring electrode separately. These two measurements are performed in the same conditions except for the existence of the sample. The complex capacitance with the sample inserted $C_{\text {tot }}$ is the parallel capacitance of air gap capacitance $C_{1}$ and the sample capacitance. The $C_{t o t}$ is given by the equation (1). The purpose of the measurement in the absence of the sample is to obtain the complex capacitances of the air $C_{\text {airref }}$ between top and bottom electrode without changing the distance between them, and then use the results of air as a reference to obtain the permittivity of the sample. In order to keep the measurement conditions same for the two measurements, the shift from the measurement with sample to that without a sample is only to remove the sample and then insert small pieces of the sample between two guard electrodes.

For deriving the permittivity of the sample, several dimension parameters are needed. The first parameter is the sample thickness $d_{\text {sample }}$ which is an average value over ten different locations. In order to avoid the deformation of the sample by the micrometer, the sample is clamped by two polycarbonate plates with the thickness of $1 \mathrm{~mm}$, and then measured by the screw micrometer. The sample thickness $d_{\text {sample }}$ is the value after subtracting the thickness of the polycarbonate. The second group of parameters are the thickness of air gaps $d_{1}$ and $d_{1}$ above or below the sample. They are the height differences between guard electrodes and measuring/high-voltage electrodes relative to the baseplate. The $d_{1}$ can be obtained by subtracting the average distance between the surface A to the surface B from the average distance between the surface $C$ to surface D in Figure 1, and the same method for $d_{2}$. The measurements of the distance between two surfaces are performed ten times over different locations by the vernier caliper or screw micrometer. The capacitance of the air gaps can be obtained if the accurate dimension parameters are given, and then the capacitance of the sample can be calculated by the equation (2). With the help of the air reference method, the complex capacitance of the sample can be derived by the equation (3).

$$
\begin{gathered}
C_{\text {tot }}=\frac{1}{1 / C_{\text {sample }}+1 / C_{1}+1 / C_{2}}=\frac{C_{\text {sample }} C_{1} C_{2}}{C_{1} C_{2}+C_{\text {sample }} C_{2}+C_{\text {sample }} C_{1}} \\
C_{\text {sample }}=\frac{1}{1 / C_{\text {tot }}-1 / C_{1}-1 / C_{2}} \\
\varepsilon_{r}^{*}=\varepsilon_{r}^{\prime}-j \varepsilon_{r}^{\prime \prime}=\frac{C_{\text {sample }}}{C_{\text {airref }} \frac{d_{\text {sample }}}{d_{1}+d_{2}+d_{\text {sample }}}}=\frac{C_{\text {sample }}}{C_{\text {airref }}} \frac{d_{1}+d_{2}+d_{\text {sample }}}{d_{\text {sample }}}
\end{gathered}
$$




\section{Measurement system}

The non-contact electrode arrangement has different geometry and potential distribution, resulting in different edge effect. Moreover, equations to derive the sample permittivity need to consider the capacitance of air gaps, probably leading to higher error sensitivity. In this chapter, the geometric influences of this arrangement are considered by FEM (finite element method) model, and the error sensitivity is compared between the non-contact and the contact measurement methods. Furthermore, the effect of the potential difference between the guard electrode and the measuring electrode is assessed.

\subsection{Edge effect}

The edge effect exists in all electrodes. The electrostatic flux tends to spread out at the edges of the electrodes and air, including a bigger area of sample than the real electrode area. This phenomenon is also called as fringing and can have an effect on the dielectric response measurements. The use of a guard ring can significantly decrease the influence of the edge effect, but this cannot be totally eliminated as the electric deformation also exists in the gap between the measuring electrode and the guard electrode. For the electrode arrangement presented in this paper, the surfaces of the high voltage electrode and the guard electrode are not in the same plane, which can probably result in a bigger error in the results of dielectric constant and the loss. Therefore, it is important to know the electric distribution for the specific electrode arrangement and obtain the correction factor for the geometric influence.

The FEM model of the electrode arrangement is established according to the geometry dimensions discussed in Chapter 1 . The applied voltage is $140 \mathrm{~V}$, the sample permittivity is 3 , the thickness of the sample is $2 \mathrm{~mm}$, and the two air gap thickness are both $0.6 \mathrm{~mm}$. The model is analyzed in Comsol Multiphysics, and the stationary study is performed to calculate the electric field distribution and the capacitance. The electric field is shown in Figure 2. It can be seen the electric field between the high voltage electrode and the measuring electrode is relatively uniform, but that between the measuring and the high-voltage electrodes is deformed, indicating additional electric flux pass through the sample and arrive at the measuring electrode. Therefore, the correction factor is needed to consider the effect of the fringing. The fringing depends on the permittivity and thickness of the sample, as well as the thickness of the electrode and air gaps. For the non-contact electrode arrangement discussed in this paper, the dimension is immobile, so the correction factors of capacitance for different sample permittivity and air gaps thickness are calculated by the FEM model, as shown in Figure 3. The influence of the sample thickness will be considered in the future work. The sum of the two gap thickness is used to consider the effect of the air gap dimensions on the results. The correction factors are the ratios of calculated capacitance to the real capacitance. It is clearly shown that the gap thickness is an important factor that makes the calculated value derived from the real value. The correction factors increase with the total thickness of air gaps, and the rising slope is higher for the samples with higher permittivity. However, the influence of edge effect is not obvious in this electrode arrangement, and the relative error is only $2.5 \%$ even the total thickness of air gaps reach $1.2 \mathrm{~mm}$ for the sample with the thickness of $1 \mathrm{~mm}$.

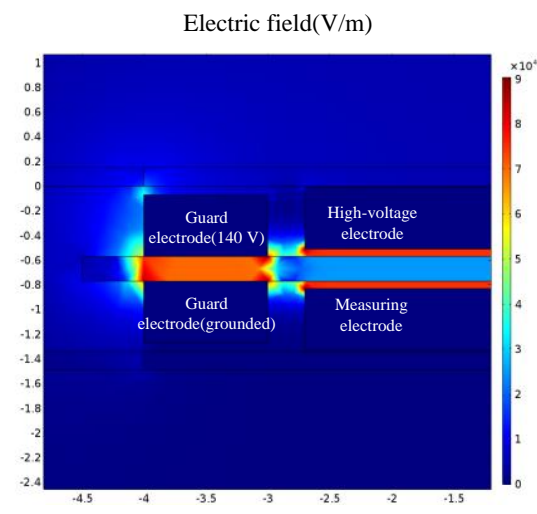

Figure 2. Sketch electric field distribution with sample permittivity of $3, d_{\text {sample }}$ is $2 \mathrm{~mm}$, and $d_{1}, d_{2}$ are both $0.6 \mathrm{~mm}$

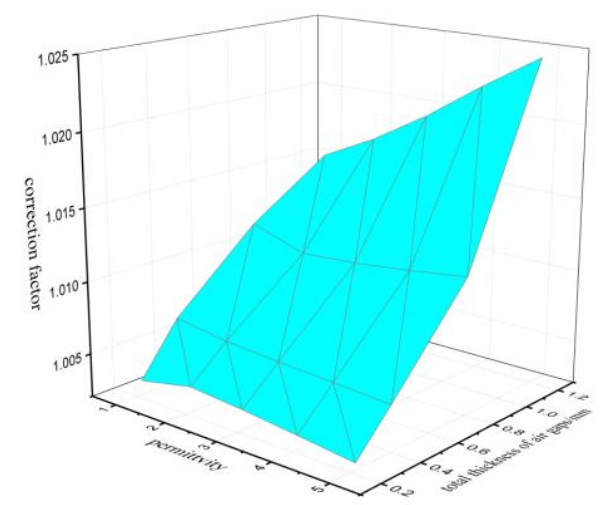

Figure 3.

Figure 3. The correction factors for different thickness air gaps and permittivity of the sample (the thickness of the sample is $1 \mathrm{~mm}$ )

\subsection{The effect of the potential difference caused by IDAX}

The IDAX is a commercial FDS measurement instrument which has been widely used in labs and field. Generally, the guard electrode (grounded) and measuring electrode are connected to the out-layer and the intermediate of the coaxial cable separately, and the out-layer and the inter-layer are connected to the two input terminals of the operational amplifier in the IDAX. For the ideal amplifier, the two input terminals are treated to be in the same potential. However, the potential between the two inputs is not absolutely zero. The small potential difference can produce a parallel capacitance with the main measurement circuit, as shown in Figure 4. If the ratio of the tested capacitance to the capacitance between the guard electrode and the electrode is not big enough, the potential difference can have an effect on the results even the potential is not significant. That could be worse if the potential between the guard and the measuring electrode has a phase shift with the applied potential, which can cause an error in the loss factor, producing more complicated error than only changing the amplitude. 


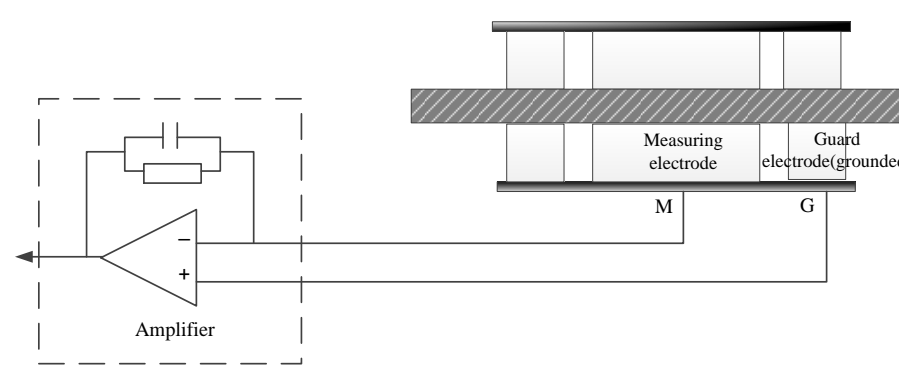

Figure 4. Sketch of the connections between the electrode and the amplifier in the IDAX300

For the specific IDAX300 in our lab, the effect of the potential between the measuring electrode and the guard electrode $U_{G M}$ is assessed by performing FDS measurement using the stable capacitances that have the same value as corresponding real capacitance. First, the capacitance between the measuring electrode and guard electrode is measured. The valued is 93.9 $\mathrm{pF}$, and two capacitances with bigger values are used in the test which are $370 \mathrm{pF}$ and $1500 \mathrm{pF}$. For this assessment, the worse situation is considered, that is when the gap between the highvoltage and the measuring electrodes is filled with air as the geometric capacitance between the two electrodes $C_{H M}$ in this situation is smallest. The distance between the high-voltage electrode and the measuring electrode is assumed to be $1.35 \mathrm{~mm}$, so the assumed capacitance between them is approximately 15 $\mathrm{pF}$. Considering the available capacitance, the value of the capacitance is multiplied by 20 , and the applied voltage is divided by 20 to be $10 \mathrm{~V}$ from $200 \mathrm{~V}$ in peak meanwhile. These two situations are equivalent in terms of assessing the effect of the potential $U_{G M}$. The related values in this measurement are shown in Table 1.

Table 1. Calculated and used capacitance in the tests

\begin{tabular}{|c|c|c|c|c|c|}
\hline $\begin{array}{c}\text { Applied } \\
\text { voltage(V) }\end{array}$ & \multicolumn{2}{|c|}{$C_{H M}(\mathrm{~F})$} & \multicolumn{3}{c|}{$C_{G M}(\mathrm{~F})$} \\
\hline 200 & \multicolumn{2}{|c|}{$1.5 \mathrm{E}-11$} & \multicolumn{3}{|c|}{$9.39 \mathrm{E}-11$} \\
\hline $\begin{array}{c}\text { Applied } \\
\text { voltage(V) }\end{array}$ & $\begin{array}{c}C_{H M} \\
(\mathrm{~F})\end{array}$ & $\begin{array}{c}C_{H M} \\
\text { substitute } \\
\text { used (F) }\end{array}$ & $C_{G M}$ & $\begin{array}{c}C_{G M} \\
\text { substitute } \\
11 \text { used (F) }\end{array}$ & $\begin{array}{c}C_{G M} \\
\text { substitute } \\
2 \text { used } \\
(\mathrm{F})\end{array}$ \\
\hline 10 & $3 \mathrm{E}-10$ & $3.02 \mathrm{E}-10$ & $9.39 \mathrm{E}-11$ & $3.7 \mathrm{E}-10$ & $1.5 \mathrm{E}-9$ \\
\hline
\end{tabular}

The results of the test are shown in Figure 5. It is found even the $U_{G M}$ is almost five times $C_{H M}$, the $C_{H M}$ has not been influenced significantly except some fluctuation in the loss. It can be concluded that the potential between the guard and measuring electrode with the IDAX300 will not have a significant effect on the FDS measurements with this electrode arrangement. It is worth noting that this potential could be significantly higher when the FDS measurements is performed with shunt, but we will not discuss it in this paper.

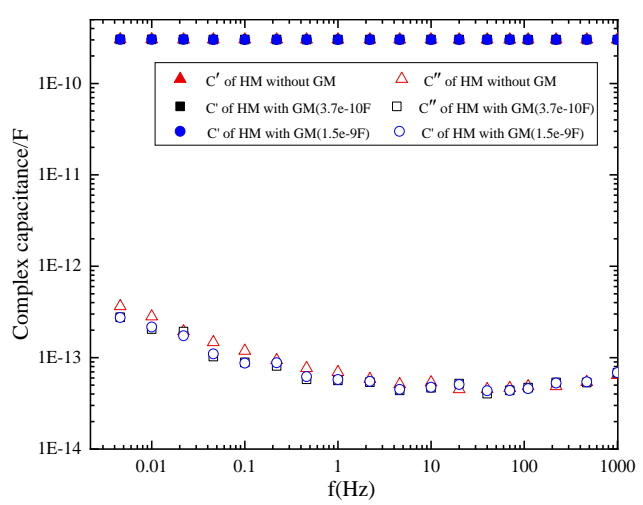

Figure 5. The effect of potential between guard and measuring electrodes on the dielectric response measurements

\subsection{Error sensitivity}

It can be speculated that a slight error can result in a much larger error in the final results by using non-contact measurements compared to contact methods due to the existence of air gaps. The error sensitivity can be quantified by analyzing the reactions of the final results to the error sources. The error sources can be divided into two categories. The first source group is the error in determining the thickness of air gaps and the sample, which has been discussed in detail in the paper [1]. Here, the other group of errors, that are measurement errors such as amplitude error, phase shift and noise, will be analyzed by considering the way IDAX perform to obtain the capacitance.

The geometric capacitance of the sample can be calculated by:

$$
C_{\text {sample }}=\varepsilon_{r} \varepsilon_{0} \frac{S}{d_{\text {sample }}}
$$

Where the $\varepsilon_{r}$ is the relative permittivity of the sample, $\varepsilon_{0}$ is the permittivity of free space, $S$ is the area of the measuring electrode, $d_{\text {sample }}$ is the thickness of the sample. In the noncontact measurements, the total geometric capacitance of the two air gaps can be presented as:

$$
\begin{gathered}
C_{\text {air }}=\varepsilon_{0} \frac{S}{d_{\text {air }}}=\varepsilon_{0} \frac{S}{d_{\text {sample }} r} \\
r=\frac{d_{\text {air }}}{d_{\text {sample }}}
\end{gathered}
$$

Where the $d_{\text {air }}$ is the total thickness of two air gaps. The geometric capacitance including the sample and air gaps can be obtained by:

$$
C_{n c}=\frac{1}{1 / C_{\text {sample }}+1 / C_{\text {air }}}=\frac{\varepsilon_{r} \varepsilon_{0} s}{d_{\text {sample }}+\varepsilon_{r} r d_{\text {sample }}}
$$

On the other hand, the geometric capacitance between the measuring electrode and the high-voltage electrode is the sample capacitance in contact measurements, as shown in (8) 


$$
C_{c}=C_{\text {sample }}=\varepsilon_{0} \varepsilon_{r} \frac{S}{d_{\text {sample }}}
$$

Here, it is assumed the amplitude error is $k_{1}$ and the phase shift is $\frac{k_{2}}{2 \pi f}$ where $f$ is the frequency of the signal, so the error factor can be represented as:

$$
K=\left(1+k_{1}\right) \cdot e^{j k_{2}}
$$

The measured currents through the sample in the contact and non-contact measurements are shown in equation (10) and equation (11) separately.

$$
\begin{aligned}
& I_{c}=j \omega C_{c} V K \\
& I_{n c}=j \omega C_{n c} V K
\end{aligned}
$$

Then the measured capacitance of the sample $C_{\text {sc_error }}$ with the effect of amplitude and phase shift errors can be obtained by equation (12), which is equal to the capacitance between the measuring electrode and the high-voltage electrode $C_{c_{-} \text {error }}$ :

$$
C_{\text {sc_error }}=C_{c_{-} \text {error }}=\frac{I_{c}}{j \omega V}=C_{c} K=C_{\text {sample }} K
$$

In the non-contact measurement, the capacitance between the measuring electrode and the high-voltage electrode can be calculated by:

$$
C_{n c_{-} \text {error }}=\frac{I_{n c}}{j \omega V}=C_{n c} K=\frac{\varepsilon_{r} \varepsilon_{0} s}{d_{\text {sample }}+\varepsilon_{r} r d_{\text {sample }}} \cdot K
$$

Then the measured capacitance of the sample considering errors can be derived to equation (14).

$$
C_{\text {snc_error }}=\frac{1}{1 / C_{n c_{-} \text {error }}-1 / C_{\text {air }}}=\varepsilon_{0} \varepsilon_{r} \frac{S}{d_{\text {sample }}} \frac{K}{r \varepsilon_{r}-K r \varepsilon_{r}+1}=C_{\text {sample }} \frac{K}{r \varepsilon_{r}-K r \varepsilon_{r}+1}
$$

The error sensitivities of contact and non-contact measurements can be compared by the following equation:

$$
\frac{C_{\text {sc_error }}}{C_{\text {snc_error }}}=\frac{C_{\text {sample }} K}{C_{\text {sample }} \frac{K}{r \varepsilon_{r}-K r \varepsilon_{r}+1}}=r \varepsilon_{r}-K r \varepsilon_{r}+1
$$

Therefore, it can be concluded that the same error in the amplitude and the phase shift can result in a larger error in final results by using non-contact measurements compared to contact measurements. With the influence of the noise, the current flows through the sample can be expressed as the equation (16) and (17). The $k_{\text {romdom }}$ in the equations is a series of random absolute amplitude error. The error sensitivity of the two types of measurements can be analyzed using the same method discussed above.

$$
I_{c}=j \omega C_{c} V+k_{\text {random }}
$$

$$
I_{n c}=j \omega C_{n c} V+k_{\text {random }}
$$

Here, it is assumed that the complex permittivity of the sample accord with the Debye distribution. The complex permittivity can be expressed as the equation (18) with the consideration of DC conductivity.

$$
\varepsilon_{\text {Debye }}^{*}=\varepsilon_{\infty}+\frac{\varepsilon_{s}-\varepsilon_{\infty}}{1+j \omega t}+\frac{\sigma_{d c}}{j \omega \varepsilon_{0}}
$$

If we assume that $\varepsilon_{\infty}=2, \varepsilon_{s}=6, t=2$, the thickness of the sample is $2 \mathrm{~mm}$, the radius of the measuring electrode is $30 \mathrm{~mm}$, and the total thickness of the air gaps in the non-contact measurements is $0.4 \mathrm{~mm}$. The complex capacitance over different frequencies is shown in Figure 6. The amplitude, phase shift and noise are added separately, and then to calculate the capacitance with the effect of the errors. For the errors, we assume $k_{1}=1 \times 10^{-2}, k_{2}=4 \times 10^{-3}$ and $k_{\text {random }}$ is a series of numbers from $-1.5 \times 10^{-12}$ to $1.5 \times 10^{-12}$. The capacitance distributions under the effect of amplitude error, phase shift error, and noise are shown in Figure 7 to Figure 9 separately. The capacitance that accord with Debye distribution is plotted in each figure for comparisons.

It is shown, the imaginary part of the complex capacitance is influenced more significantly in low frequencies than in high frequencies. The results obtained by the means of the noncontact method derive more from the real value than the results obtained by the means of contact method.

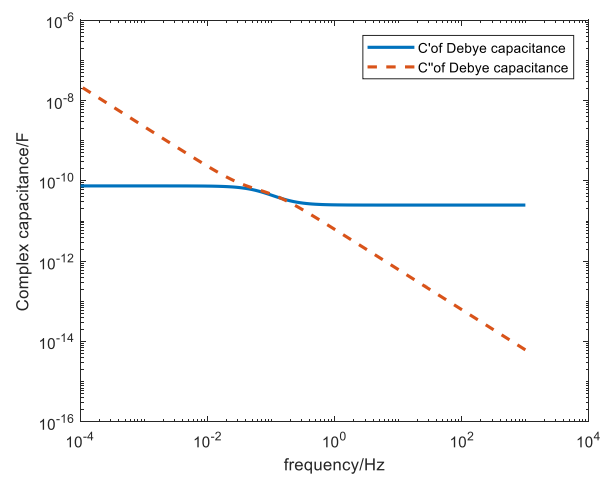

Figure 6. Complex capacitance that accord with Debye distribution

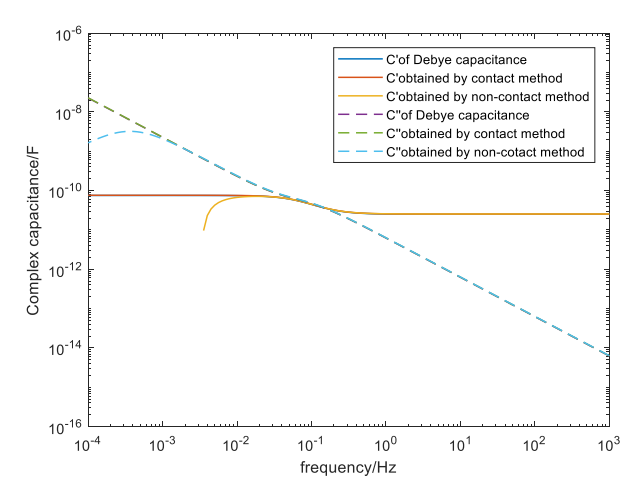

Figure 7. Complex capacitance distributions under the effect of amplitude error 


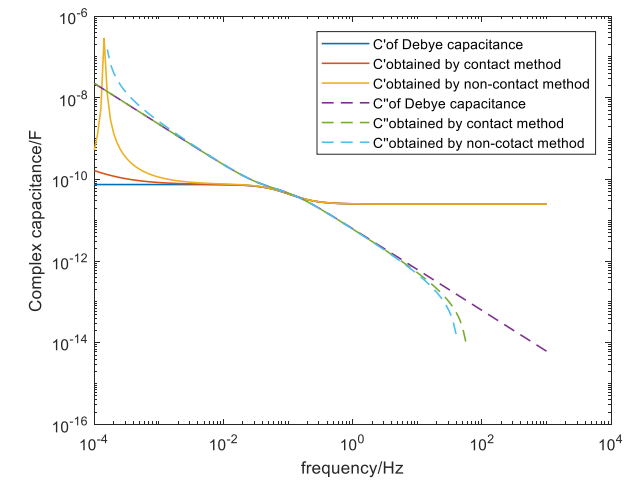

Figure 8. Complex capacitance distributions under the effect of phase shift error

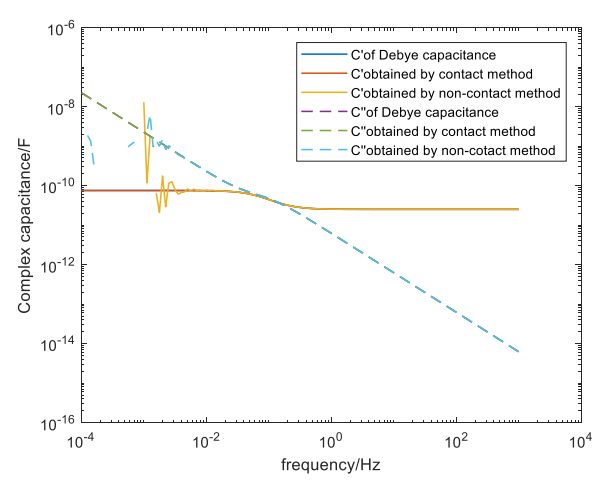

Figure 9. Complex capacitance distributions under the effect of noise

\section{Conclusions}

In this paper, one type of electrode arrangement that can avoid direct contacts of the sample with both the bottom and the top electrode is introduced. The edge effect is calculated by the FEM model. The air reference method is used to eliminate the influence of system errors. The measurement instrument is described and the influence of the potential difference between two terminals of IDAX input is analyzed. The error sensitivities are compared between the non-contact and contact methods. Overall, this non-contact electrode arrangement combined with the IDAX 300 can potentially improve the accuracy of dielectric response measurements although this method can increase the sensitivities to errors. The relations between the gap thickness and sample properties (permittivity, thickness) can significantly influence the reliability and the accuracy of the non-contact measurements with the consideration of the precision of the IDAX and the edge effect, which will be analysed in our future work.

\section{References}

[1] X. Xu, T. Bengtsson, and J. Blennow, "Enhanced accuracy in dielectric response material characterization by air reference method", IEEE Transactions on Dielectrics \& Electrical Insulation, 20. 3., 2013, pp. 913-921.

[2] P. S. Ho, P. O. Hahn, J. W. Bartha, G. W. Rubloff, F. K. LeGoues, and B. D, "Silverman. Chemical Bonding and Reaction at Metal/Polymer Interfaces", Journal of Vacuum Science \& Technology A: Vacuum Surfaces \& Films, 3. 3., 1985, pp. 739-745.
[3] T. I. Jones, "Mercury electrodes for measurements on solid dielectrics at radio frequencies", Electrical Engineers Journal of the Institution, 74. 446., 1934, pp. 179-186.

[4] J. W. Lane, J. C. Seferis, and M. A, "Bachmann, Dielectric studies of the cure of epoxy matrix systems", Journal of Applied Polymer Science, 31. 5., 2010, pp. 1155-1167.

[5] M. T. Tlili, S. Ghodhbane, and J. Dhahri, "Influence of temperature and Prdoping on the dielectric properties of $\mathrm{Ca}_{2-x} \mathrm{Pr}_{x} \mathrm{MnO}_{4}$ compounds and structural transition effects", Phase Transitions, 90. 10., 2017, pp. 964-973.

[6] R. Richert, "Insulated electrodes for eliminating conductivity in dielectric relaxation experimentsm", European Physical Journal B, 68. 2., 2009, pp. 197200.

[7] A. V. Vykhodtsev, B. Kordi and D. R. Oliver, "Sensitivity analysis of a parallel-plate method for measuring the dielectric permittivity of high-voltage insulating materials", High Voltage, 2. 3., 2017, pp. 200-204.

[8] Y. Zhou, C. Zhang, W. Li, Z. Xing and W. Yang, "Correction of contactfree measurement in outdoor insulation diagnostics," In Proc. 2016 International Conference on Condition Monitoring and Diagnosis, Xi'an, 2016, pp. 360-363.

[9] X. Xu, T. Bengtsson, J. Blennow, and S. M. Gubanski, "Correction of Geometric influence in permittivity determination," In Proc. Nordic Insulation Symposium, Trondheim, 2011, pp. 71-75.

[10] C. Yuan, C. Xie, L. Li, X. Xu, S. M. Gubanski, and Z. He, "Dielectric response characterization of in-service aged sheds of (U) HVDC silicone rubber composite insulators," IEEE Transactions on Dielectrics and Electrical Insulation, 23. 3., 2016, pp. 1418-1426. 